\title{
RESEARCH
}

Open Access

\section{Effect of direct-acting antivirals on corrected QT interval and cardiac functions in patients with chronic hepatitis $C$ virus infection}

Mohamed Gamal Ibrahim', Ahmed Abdelrahman Sharafeldin' , Nevine Ibrahim Mousa ${ }^{2}$, Tarek Khairy Mousa ${ }^{1}$ and Ahmed Mohamed El Missiri ${ }^{1 *}$ (D)

\begin{abstract}
Background: Hepatitis C virus (HCV) infection is a major public health problem in Egypt. The use of direct-acting antivirals (DAAs) in such patients has been shown to be highly effective. The cardiac safety of such antivirals remains uncertain. This study aimed to assess the effect of the novel DAAs on corrected QT (QTC) interval and on cardiac function using trans-thoracic echocardiography.

Results: This was a prospective cohort study performed on 100 patients suffering from chronic HCV infection. Patients were into two equal groups according to the presence of liver cirrhosis. The group without liver cirrhosis received a daily combination of sofosbuvir $400 \mathrm{mg}$ and daclatasvir $60 \mathrm{mg}$ for 12 weeks while that with liver cirrhosis (Child-Pugh score A or B) received a daily combination of sofosbuvir 400 mg, daclatasvir 60 mg, and ribavirin 600 $\mathrm{mg}$ for 12 weeks. Surface ECG and trans-thoracic echocardiography were performed prior to the start of treatment and after 12 weeks of treatment.

At the end of treatment, no changes were observed in QTC interval in those with $(p=0.48)$ or without $(p=0.048)$ liver cirrhosis. In patients without liver cirrhosis, right ventricular global longitudinal strain (RV GLS) decreased from $22(-30$ to -17$)$ to $-21(-27-18), p=0.024$. In patients with liver cirrhosis, lateral mitral $E^{\prime}$ velocity was reduced from $14.38 \pm 3.59$ to $13.62 \pm 3.21 \mathrm{~cm} / \mathrm{s}, p=0.02$ and indexed left atrial volume (LAVI) was increased from $25.96 \pm 3.96$ to $26.86 \pm 4.12 \mathrm{ml} / \mathrm{m}^{2}, p=0.032$. There were no changes in both groups regarding left ventricular (LV) dimensions, ejection fraction, trans-mitral E/A ratio, E/E' ratio, deceleration time, right ventricular (RV) systolic pressure, mean pulmonary artery pressure, RV fractional area change, tricuspid annular plane systolic excursion, and LV GLS.

Conclusion: The current national protocol of HCV infection treatment with direct-acting antiviral agents used in Egyptian patients has a good cardiac safety profile. Such treatments have no effect on QTc interval, left and right ventricular functions except for a decrease in RV GLS in those with no liver cirrhosis and a reduction in lateral mitral $\mathrm{E}^{\prime}$ velocity in those with liver cirrhosis both remained within the normal reference range.
\end{abstract}

Keywords: Echocardiography, Hepatitis C virus, Direct-acting antiviral, Cardiac function

\footnotetext{
*Correspondence: amissiri@med.asu.edu.eg; amissiri@yahoo.com

${ }^{1}$ Cardiology Department, Ain Shams University, Abbassia square, Abbasia,

Cairo 11566, Egypt

Full list of author information is available at the end of the article
} 


\section{Background}

Hepatitis $\mathrm{C}$ virus (HCV) infection is a major cause of chronic liver disease worldwide. The long-term impact of $\mathrm{HCV}$ infection varies from minimal histological changes to extensive fibrosis and cirrhosis with or without hepatocellular carcinoma [1].

Although $\mathrm{HCV}$ is primarily a hepatotropic virus, it may lead to extra-hepatic manifestations. HCV was isolated from the myocardium of patients suffering from myocarditis and cardiomyopathy and is therefore additionally considered a cardiotropic virus [2].

It has been shown that up to $50 \%$ of patients with advanced liver cirrhosis may have cardiac dysfunction, a condition often called cirrhotic cardiomyopathy. Such patients have normal or increased cardiac output at rest with a blunted response to stress. They may also have QT interval prolongation and chronotropic incompetence [3].

Treatment of chronic $\mathrm{HCV}$ infection evolved from the limited efficacy interferon-based therapy to the highly effective direct-acting antiviral (DAA) agents. Novel DDAs have received breakthrough therapy status by the Food and Drug Administration; however, retrospective studies, case reports, and post-marketing reports suggest possible deleterious effects to the heart including bradyarrhythmia and toxic cardiomyopathy $[4,5]$.

Advances in trans-thoracic echocardiography with the application of strain analysis and speckle tracking provide sensitive assessment of cardiac function that may predict clinical outcomes in various heart diseases, including those with $\mathrm{HCV}$ infection $[6,7]$.

The aim of this study was to assess the effect of treatment of chronic HCV infection with DAAs on corrected QT interval (QTc) of the surface ECG and on cardiac function using trans-thoracic echocardiography.

\section{Methods}

The study protocol received approval from the institutional ethical committee. Written informed consents were provided by all participants. A prospective cohort study was conducted on 100 treatment-naïve adult patients $(\geq$ 18 years old) suffering from chronic HCV infection-confirmed by a polymerase chain reaction test for $\mathrm{HCV}$ (genotype 4). Patients were randomly selected from the institution's specialized clinic for HCV-infected patients in the period from March 2018 to September 2019.

Patients were excluded from the study if they had a history of arrhythmias or a current arrhythmia; any type of cardiomyopathy; coronary heart diseases; valvular heart disease; uncontrolled hypertension; previously treated chronic HCV patients; concomitant infection with either of hepatitis $B$ virus, human immunodeficiency virus or bilharziasis; patients with an estimated glomerular filtration rate less than $30 \mathrm{ml} / \mathrm{min}$; pregnant women; those with any form of autoimmune diseases; those with a history of malignancy; and those receiving medications known to be cardiotoxic, have a negative chronotropic or antiarrhythmic effect.

Patients were assigned into two equal groups (50 each) depending on the presence of liver cirrhosis (defined as Child-Pugh score A and B) [8]. All patients were treated according to the national protocol for the management of chronic HCV infection [9, 10] as follows: those without liver cirrhosis received a combination of sofosbuvir $400 \mathrm{mg}$ daily and daclatasvir $60 \mathrm{mg}$ daily for 12 weeks. Those with liver cirrhosis received a combination of sofosbuvir $400 \mathrm{mg}$ daily, daclatasvir $60 \mathrm{mg}$ daily and ribavirin $600 \mathrm{mg}$ daily for 12 weeks.

\section{Clinical history and baseline investigations}

Detailed patient history was taken specially to define Child-Pugh score and to assess for the presence of exclusion criteria. Height in centimeters and weight in kilograms were measured to estimate body surface area (BSA) in $\mathrm{m}^{2}$. BSA was calculated using the Mosteller formula where $\mathrm{BSA}=$ square root of ([height in $\mathrm{cm} \times$ weight in $\mathrm{kg}] / 3600)$ [11].

Venous blood samples were obtained from all patients to measure serum albumin, total bilirubin, prothrombin time, partial thromboplastin time, international normalized ratio, liver enzymes, rapid ELISA testing of $\mathrm{HCV}$ antibody and hepatitis B surface antigen. Additionally, quantitative PCR of HCV antibody was assessed before the start of treatment, at 4 weeks, at the end of treatment, and 12 weeks after the end of treatment. Abdominal ultrasonography was performed at baseline to examine for criteria of liver cirrhosis, as well as, Fibroscan assessment of liver stiffness/scaring.

\section{Cardiac investigations}

The following was performed at baseline and 1 week after the end of treatment:

12-lead surface ECG: to assess heart rate, presence of any degree of heart block, and to assess corrected QT interval.

Standard trans-thoracic echocardiography: standard transthoracic echocardiography with machine-integrated ECG recording was performed for all patients using an S7 machine with an M4S matrix sector array probe with a frequency range from 1.7 to $342 \mathrm{MHz}$ (GE Vingmed, Horten, Norway). A standard study following was performed for all patients by an echocardiographer accredited by the European Association of Cardiovascular Imaging (EACVI) following guideline recommended protocols [12] to obtain the following measurements: Left ventricular end-diastolic diameter (LVEDD) and left ventricular end-systolic diameter (LVESD) measured by M-mode from the parasternal short-axis view at the level of the papillary muscles; left ventricular enddiastolic volume (LVEDV), end-systolic volume (LESV), and ejection fraction (LVEF) measured by the modified 
Simpson's method [12]; LV volumes were then indexed according to BSA to calculate indexed LVEDV (LVEDVI) and indexed LVESV (LVESVI). Indexed left atrial volume (LAVI) was measured using the biplane Simpson's method from the apical 4- and 2-chamber views [12].

LV diastolic function was assessed by estimating transmitral E/A ratio, deceleration time (DT), lateral mitral annulus tissue Doppler first negative wave velocity (E'), and $\mathrm{E} / \mathrm{E}$ ' ratio.

Right ventricular (RV) function was assessed by estimating mean pulmonary artery pressure (PAP), RV systolic pressure (RVSP), tricuspid annular plane systolic excursion (TAPSE), and RV fractional area change (RVFAC). The latter was calculated as (RV end-diastolic area-RV end-systolic area)/RV end-diastolic area $\times 100$ [13].

2-D left ventricular strain: analysis of digital loops acquired from the apical 2-, 3-, and 4-chamber views was performed using machine-integrated software for speckle tracking to calculate the peak global longitudinal strain (GLS) [14].

2-D right ventricular strain: analysis of an RV-focused apical 4-chamber view digital loop was performed using speckle tracking for off-line semi-automated analysis of the speckle-based RV free wall longitudinal strain [15].

\section{Statistics}

Data were statistically analyzed using IBM SPSS Statistics version 25 (IBM corporation, Armonk, NY, U.S.). Categorical variables were expressed as number and percentage and analyzed using the chi-square test. Continuous variables were expressed as mean $\pm \mathrm{SD}$ and analyzed using Student's $t$ test for variables that passed normality tests and Mann-Whitney $U$ test for those that did not pass normality. Correlations were analyzed using Pearson's correlation coefficient $(r)$. A probability value $p<$ 0.05 was considered statistically significant and a $p$ value $<0.0001$ was considered highly significant.

\section{Results}

\section{Baseline characteristics}

Baseline demographic and clinical characteristics, as well as assessment of liver status by abdominal ultrasonography and fibroscan, are shown in Table 1 . Patients in the liver cirrhosis group were older $52 \pm 12.04$ versus $46.76 \pm 12.1$ years, $p=0.032$. Additionally, there was a higher percentage of diabetics in the liver cirrhosis group 14 (28\%) versus 7 (14\%), $p=0.086$.

\section{Effect of treatment on cardiac function in patients with no liver cirrhosis}

Treatment with direct-acting antivirals in patients with no liver cirrhosis caused a significant reduction in RV GLS from $22(-30$ to -17$)$ to $-21(-27-18), p=0.024$. However, treatment had no effect on QTc interval, LV systolic and diastolic functions, and no effect on other RV functions as shown in Table 2.

\section{Effect of treatment on cardiac function in patients with liver cirrhosis}

Child-Pugh score's distribution in patients with liver cirrhosis was A5 $=31$ (62\%), A6 $=7(14 \%)$, and B7 $=12$ (24\%) patients.

Table 1 Comparing demographic and clinical characteristics

\begin{tabular}{|c|c|c|c|}
\hline Variable & No cirrhosis $(n=50)$ & Cirrhosis $(n=50)$ & $p$ value \\
\hline \multicolumn{4}{|l|}{ Clinical characteristics } \\
\hline Age, years & $46.76 \pm 12.1$ & $52 \pm 12.04$ & 0.032 \\
\hline Male gender, $n(\%)$ & $19(38 \%)$ & $21(42 \%)$ & 0.683 \\
\hline Current smoker, $n(\%)$ & $11(22 \%)$ & $11(22 \%)$ & 1 \\
\hline Hypertension, $n(\%)$ & $13(26 \%)$ & $28(56 \%)$ & 0.002 \\
\hline Diabetes, $n(\%)$ & $7(14 \%)$ & $14(28 \%)$ & 0.086 \\
\hline \multicolumn{4}{|c|}{ Liver assessment by abdominal ultrasound at baseline } \\
\hline Normal & $40(80 \%)$ & $0(0 \%)$ & $<0.0001$ \\
\hline Parenchymatous liver disease & $10(20 \%)$ & $38(76 \%)$ & \\
\hline Cirrhotic liver disease & $0(0 \%)$ & $12(24 \%)$ & \\
\hline \multicolumn{4}{|l|}{ Fibroscan assessment at baseline } \\
\hline Fo & $13(26 \%)$ & $0(0 \%)$ & $<0.0001$ \\
\hline F1 & 27 (54\%) & $0(0 \%)$ & \\
\hline F2 & $9(18 \%)$ & $0(0 \%)$ & \\
\hline F3 & $1(2 \%)$ & 16 (32\%) & \\
\hline F4 & $0(0 \%)$ & 34 (68\%) & \\
\hline
\end{tabular}


Table 2 Comparing ECG and echocardiographic variables in patients with no liver cirrhosis before and after treatment

\begin{tabular}{|c|c|c|c|}
\hline Variable & Before treatment $(n=50)$ & After treatment $(n=50)$ & $p$ value \\
\hline QTc, ms & $406.64 \pm 18.73$ & $408.76 \pm 14.12$ & 0.48 \\
\hline LVEDD, mm & $47.36 \pm 3.29$ & $47.84 \pm 2.57$ & 0.26 \\
\hline LVESD, mm & $29.76 \pm 2.81$ & $29.6 \pm 1.96$ & 0.701 \\
\hline LVEDV, ml & $101.08 \pm 19.01$ & $103.6 \pm 17.15$ & 0.183 \\
\hline LVESV, ml & $54.18 \pm 9.40$ & $55.48 \pm 8.69$ & 0.205 \\
\hline LVEDVI, $\mathrm{ml} / \mathrm{m}^{2}$ & $34.48 \pm 7.19$ & $33.24 \pm 6.21$ & 0.133 \\
\hline LVESVI, $\mathrm{ml} / \mathrm{m}^{2}$ & $18.43 \pm 3.79$ & $17.88 \pm 3.08$ & 0.224 \\
\hline LVEF, \% & $57.3 \pm 3.71$ & $57.54 \pm 4.15$ & 0.268 \\
\hline $\mathrm{LAVI}, \mathrm{ml} / \mathrm{m}^{2}$ & $25.38 \pm 4.69$ & $25.98 \pm 4.13$ & 0.174 \\
\hline Trans-mitral E/A ratio & $1.21 \pm 0.38$ & $1.18 \pm 0.32$ & 0.323 \\
\hline DT, ms & $200.08 \pm 31.09$ & $204.84 \pm 30.49$ & 0.285 \\
\hline Lateral mitral $\mathrm{E}^{\prime}, \mathrm{cm} / \mathrm{s}$ & $15.2 \pm 4.52$ & $15.7 \pm 3.47$ & 0.250 \\
\hline $\mathrm{E} / \mathrm{E}^{\prime}$ ratio & $6.32 \pm 2.31$ & $6.3 \pm 1.54$ & 0.373 \\
\hline RVSP, $\mathrm{mmHg}$ & $25.52 \pm 5.84$ & $24.96 \pm 5.29$ & 0.474 \\
\hline Mean PAP, mmHg & $15.28 \pm 3.68$ & $15.28 \pm 3.69$ & 1 \\
\hline TAPSE, mm & $24.06 \pm 2.65$ & $24.86 \pm 2.94$ & 0.052 \\
\hline RVFAC, \% & $45.64 \pm 4.89$ & $46.46 \pm 5.13$ & 0.120 \\
\hline LV GLS & $-20(-28$ to -17$)$ & $-20(-24$ to -17$)$ & 0.161 \\
\hline RV GLS & $-22(-30$ to -17$)$ & $-21(-27$ to -18$)$ & 0.024 \\
\hline
\end{tabular}

Continuous variables for variables that passed normality tests are expressed as mean and standard deviation while for those that did not pass normality test as median (range).QTc indicated corrected QT interval, LVEDD indicates left ventricular end-diastolic dimension, LVESD indicates left ventricular end-systolic dimension, LVEDV indicates left ventricular end-diastolic volume, LVESV indicates left ventricular end-systolic volume, LVEDVI indicates indexed left ventricular end-diastolic volume, indexed LVESVI indicates left ventricular end-systolic volume, LVEF indicates left ventricular ejection fraction, LAVI indicated indexed left atrial volume, RVSP indicated right ventricular systolic pressure, PAP indicated pulmonary artery pressure, TAPSE indicates tricuspid annular plane systolic excursion, RVFAC indicates right ventricular fractional area change, LV GLS indicates left ventricular global longitudinal strain, RV GLS indicates right ventricular global longitudinal strain

Treatment with direct-acting antivirals in patients with liver cirrhosis caused a reduction in lateral mitral E' from $14.38 \pm 3.59$ to $13.62 \pm 3.21 \mathrm{~cm} / \mathrm{s}, p=0.02$ and an increase in LAVI from $25.96 \pm 3.96$ to $26.86 \pm 4.12 \mathrm{ml} /$ $\mathrm{m}^{2}, p=0.032$. However, treatment had no effect on QTc interval, other measures of LV systolic and diastolic functions, and RV functions as shown in Table 3.

\section{Discussion}

$\mathrm{HCV}$ infection is a major cause of chronic liver diseases and liver cirrhosis worldwide. It is associated with numerous hepatic and extra-hepatic manifestations including cardiac affection $[1,2]$. Novel treatment regimens in the form of direct-acting antivirals that target different steps in the HCV lifecycle have proven to be effective. However, their cardiac safety remains in question [5].

This study aimed to assess the effect of treatment of chronic $\mathrm{HCV}$ infection with DAAs used in the national protocol for the management of chronic HCV infection on QTc interval of the surface ECG and on cardiac function using trans-thoracic echocardiography. All patients were treatment-naïve with 50 patients without liver cirrhosis and 50 patients with liver cirrhosis (Child-Pugh score $\mathrm{A}$ and $\mathrm{B})$.

The main findings of this study are that DAAs used in the national Egyptian treatment protocol for HCV infection treatment have no effect on QTc interval in both patients with and without liver cirrhosis. RV GLS decreased in those with no liver cirrhosis after treatment while lateral mitral E' velocity was reduced and LAVI was increased in those with liver cirrhosis after treatment with all measurements remaining within the normal reference range. Additionally, these agents have no effect on left and right ventricular dimensions, volumes, other systolic, and diastolic functions as assessed by the various guideline-recommended measurements [12-15]. To the best of our knowledge, this is the first study to assess RV GLS in HCV patients treated with DAAs.

Baseline patient characteristics showed patients in the group with cirrhosis to be older which is expected as cirrhosis is part of disease progression that occurs over time. Additionally, hypertension was more common in those with liver cirrhosis. This could be attributed to advanced age and the pathological changes that occur with progressive liver cirrhosis. A study by Novo et al. in 
Table 3 Comparing ECG and echocardiographic variables in patients with liver cirrhosis before and after treatment

\begin{tabular}{|c|c|c|c|}
\hline Variable & Before treatment $(n=50)$ & After treatment $(n=50)$ & $p$ value \\
\hline QTc, ms & $415.84 \pm 24.40$ & $410.96 \pm 29.41$ & 0.48 \\
\hline LVEDD, mm & $48.14 \pm 2.81$ & $49.3 \pm 2.33$ & 0.26 \\
\hline LVESD, mm & $29.8 \pm 2.44$ & $30.18 \pm 2.04$ & 0.701 \\
\hline LVEDV, ml & $94.96 \pm 18.67$ & $96.84 \pm 17.71$ & 0.183 \\
\hline LVESV, ml & $31.16 \pm 6.51$ & $31.92 \pm 5.32$ & 0.359 \\
\hline LVEDVI, $\mathrm{ml} / \mathrm{m}^{2}$ & $52.98 \pm 9.08$ & $54.14 \pm 9.59$ & 0.133 \\
\hline LVESVI, $\mathrm{ml} / \mathrm{m}^{2}$ & $17.4 \pm 3.01$ & $17.82 \pm 2.8$ & 0.354 \\
\hline LVEF, \% & $66.04 \pm 2.98$ & $65.42 \pm 4.9$ & 0.41 \\
\hline $\mathrm{LAVI}, \mathrm{ml} / \mathrm{m}^{2}$ & $25.96 \pm 3.96$ & $26.86 \pm 4.12$ & 0.032 \\
\hline Trans-mitral E/A ratio & $1.09 \pm 0.38$ & $1.1 \pm 0.36$ & 0.678 \\
\hline DT, ms & $182.36 \pm 30.85$ & $180.98 \pm 21.85$ & 0.751 \\
\hline Lateral mitral $\mathrm{E}^{\prime}, \mathrm{cm} / \mathrm{s}$ & $14.38 \pm 3.59$ & $13.62 \pm 3.21$ & 0.02 \\
\hline $\mathrm{E} / \mathrm{E}^{\prime}$ ratio & $6.77 \pm 2.44$ & $6.61 \pm 2.22$ & 0.62 \\
\hline RVSP, $\mathrm{mmHg}$ & $26.34 \pm 6.06$ & $27.54 \pm 7.15$ & 0.131 \\
\hline Mean PAP, mmHg & $16 \pm 3.1$ & $16.86 \pm 3.47$ & 0.097 \\
\hline TAPSE, mm & $24.56 \pm 3.08$ & $23.94 \pm 2.8$ & 0.164 \\
\hline RVFAC, \% & $45.72 \pm 4.88$ & $44.98 \pm 4.86$ & 0.149 \\
\hline LV GLS & $-20(-26--16.5)$ & $-20(-24--16)$ & 0.198 \\
\hline RV GLS & $-22(-30--17)$ & $-21(-30--16)$ & 0.122 \\
\hline
\end{tabular}

Continuous variables for variables that passed normality tests are expressed as mean and standard deviation while for those that did not pass normality test as median (range).QTc indicated corrected QT interval, LVEDD indicates left ventricular end-diastolic dimension, LVESD indicates left ventricular end-systolic dimension, LVEDV indicates left ventricular end-diastolic volume, LVESV indicates left ventricular end-systolic volume, LVEDVI indicates indexed left ventricular end-diastolic volume, indexed LVESVI indicates left ventricular end-systolic volume, LVEF indicates left ventricular ejection fraction, LAVI indicated indexed left atrial volume, RVSP indicated right ventricular systolic pressure, PAP indicated pulmonary artery pressure, TAPSE indicates tricuspid annular plane systolic excursion, RVFAC indicates right ventricular fractional area change, LV GLS indicates left ventricular global longitudinal strain, RV GLS indicates right ventricular global longitudinal strain

2018 on 39 patients with HCV-induced liver cirrhosis without previous cardiovascular events and 39 controls without liver or cardiovascular disease examined the early features of cardiovascular damage in HCVcompensated cirrhotic patients treated with DAAs using myocardial deformation indices. In this study, both diabetes mellitus and hypertension were more common among cirrhotic patients [16].

As mentioned, QTc interval did not change after treatment in this study. This was investigated by others. In 2017, Biomy et al. investigated the cardiovascular effects of DAAs in 170 patients with HCV infection. They divided their patients into two groups: group $1(n=100)$ received pegylated interferon alfa, sofosbuvir, and ribavirin while group $2(n=70)$ received sofosbuvir and simeprevir. After 6 to 12 months of follow-up, their results showed that there was no change in the QTc interval. Additionally, no arrhythmias were observed throughout the study and during follow-up visits [17]. Also, in 2017 Durante-Mangoni et al. examined the ECG of $39 \mathrm{HCV}$ infection patients treated with either a sofosbuvir- $(n=$ $26)$ or a non-sofosbuvir-based treatment $(n=13)$. ECG tracings were obtained on the first day of treatment then after 7,14 , and 28 days. Their results showed that for the sofosbuvir group QTc interval significantly increased at 1 week $(p=0.013)$ then returned to baseline values later during therapy till the end of treatment [18].

Minor changes occurred in a couple of measurements of diastolic function in patients from the current study who had liver cirrhosis with lateral mitral E` being reduced and LAVI increased. All other diastolic parameters did not change after treatment. In the study by Novo et al. lateral E' velocity decreased after treatment $(p=0.001)$ [16]. However, in that by Biomy et al., no changes occurred in E/A ratio, DT, and E/E' [17].

The current study showed no changes in LV volumes and EF after treatment in both patients with and without liver cirrhosis. This was evaluated in 2018 in a study by El-Adawy et al. that examined the effect of different $\mathrm{HCV}$ infection treatment regimens on the heart using cardiac magnetic resonance imaging (CMR) and creatine kinase MB fraction (CK-MB) in 390 patients. Patients were divided into four groups according to the treatment regimen. Group A was treated with ledipasvir and sofosbuvir (LED + SOF), group B was treated with simepriver and sofosbuvir (SIM $+\mathrm{SOF}$ ), group $\mathrm{C}$ was treated with 
sofosbuvir and daclatasvir (SOF + DCV), and group D was treated with sofosbuvir, daclatasvir, and ribavirin $(\mathrm{SOF}+\mathrm{DCV}+\mathrm{RBV})$. CMR was done before starting treatment, during follow-up and after 6 months of treatment. Groups C and D showed a significant elevation of CK-MB levels with increased edema, early and late gadolinium enhancement compared with group B. These changes were fully reversible after the stoppage of treatment without leaving any permanent cardiac damage [19]. In concordance with the current study, the studies by Novo et al. [16] and Biomy et al. [17] showed no differences in LV dimensions, volumes, and EF before and after treatment.

RV echocardiographic parameters such as TAPSE, RVFAC, RVSP, and mean PAP showed no change after treatment. Biomy et al. had similar findings [17] while Novo et al. agreed except for TAPSE which was markedly reduced after treatment in their study $(p<0.01)$ [16].

Regarding GLS assessment of the left and right ventricles in the current study, only RV GLS showed a significant reduction after treatment in patients without liver cirrhosis. A study in 2018 by Mazzitelli et al. measured possible changes in cardiovascular function in 82 patients with chronic HCV infection before and after sofosbuvir-based regimens using both LVEF and LV GLS Interestingly, LV GLS in noncirrhotic patients improved from $-20.8 \%$ at baseline to $-21.4 \%$ at 1 month then worsened to $-20.3 \%$ at the end of treatment $(p=0.031)$ [20]. On the other hand, Novo et al. found no changes in LV GLS after treatment [16].

We could only find one study that measured RV GLS using speckle tracking echocardiography in 49 patients with liver cirrhosis and 34 healthy controls. They concluded that RV GLS had a negative correlation to cirrhosis severity (defined as Child-Pugh score) $(r=-0.60, p<0.0001)$ [21]. However, no previous study examined the effect of treatment with DAAs on RV GLS.

The reduction in the RV GLS only in those without liver cirrhosis could be explained by the changes in systemic hemodynamics caused by liver cirrhosis. Patients with liver cirrhosis have a hyperdynamic circulation with increased cardiac output and heart rate, large blood volume, low total peripheral resistance, and low or normal blood pressure [22-24]. Additionally, those with liver cirrhosis received ribavirin $600 \mathrm{mg}$ daily in addition to the DAAs used in both groups in accordance with the national protocol of management of HCV infection. Ribavirin is known to cause significant anemia which in turn has an effect on systemic hemodynamics [25]. Such different hemodynamics between those with and without liver cirrhosis could explain the difference.

\section{Study limitations}

Limitations of the current study are that it comes from a single medical center, follow-up was performed only once 1 week after the end of treatment, patients with underlying cardiac conditions were excluded. The use of ribavirin in the patients with liver cirrhosis may be considered a confounding factor; however, we had to abide by the national treatment protocols.

\section{Conclusion}

The current national protocol of HCV infection treatment with direct-acting antiviral agents used in Egyptian patients has a good cardiac safety profile. Such treatments have no effect on QTc interval, left and right ventricular functions except for a decrease in RV GLS in those with no liver cirrhosis and a reduction in lateral mitral E' velocity in those with liver cirrhosis both remained within the normal reference range.

\section{Abbreviations}

BSA: Body surface area; DAA: Direct-acting antivirals; DT: Deceleration time; EACVI: European Association of Cardiovascular Imaging;

ECC: Electrocardiogram; ELISA: Enzyme linked immunosorbent assay; FAC: Fractional area change; GE: General Electric; GLS: Global longitudinal strain; HCV: Hepatitis C virus; LAVI: Indexed left atrial volume; LV: Left ventricle; LVEDD: Left ventricular end-diastolic diameter; LVEDV: Left ventricular end-diastolic volume; LVEDVI: Indexed left ventricular enddiastolic volume; LVEF: Left ventricular ejection fraction; LVESD: Left ventricular end-systolic diameter; LVESV: Left ventricular end-systolic volume; LVESVI: Indexed left ventricular end-systolic volume; MHz: Megahertz; PAP: Pulmonary artery pressure; PCR: Polymerase chain reaction; QTc: Corrected QT interval; RV: Right ventricle; RVSP: Right ventricular systolic pressure; TAPSE: Tricuspid annular plane systolic excursion

\section{Acknowledgements}

Not applicable.

\section{Authors' contributions}

MGl collected, analyzed and interpreted the patient data and was a major contributor in writing the manuscript. AAS revised the data set. NI revised the data set and designed the antiviral treatment protocol. TKM revised the data set. AME analyzed and interpreted the patient data, and was a major contributor in writing the manuscript. All authors read and approved the final manuscript.

\section{Funding}

Not applicable.

\section{Availability of data and materials}

The datasets used and analyzed during the current study are available from the corresponding author on reasonable request.

\section{Ethics approval and consent to participate}

Approval of Ain Shams University ethical committee was obtained for this study (Committee reference number: not applicable). Written informed consents were provided by all participants.

\section{Consent for publication}

Not applicable.

\section{Competing interests}

The authors declare that they have no competing interests.

\section{Author details}

${ }^{1}$ Cardiology Department, Ain Shams University, Abbassia square, Abbasia, Cairo 11566, Egypt. ²Department of Internal Medicine, Gastroenterology and 
Hepatology, Ain Shams University, Abbassia square, Abbasia, Cairo 11566, Egypt.

Received: 26 September 2019 Accepted: 30 January 2020

Published online: 07 February 2020

\section{References}

1. Messina JP, Humphreys I, Flaxman A et al (2014) Global distribution and prevalence of hepatitis $C$ virus genotypes. Hepatology 61:77-87

2. Sanchez MJ, Bergasa NV (2008) Hepatitis C associated cardiomyopathy: potential pathogenic mechanisms and clinical implications. Med Sci Monit 14:RA55-RA63

3. Zardi EM, Abbate A, Zardi DM et al (2010) Cirrhotic Cardiomyopathy. J Am Coll Cardiol 56:539-549

4. Petta S, Maida M, Macaluso FS et al (2016) Hepatitis C virus infection is associated with increased cardiovascular mortality: a meta-analysis of observational studies. Gastroenterology 150:145-155

5. Sherman RE, Li J, Shapley S et al (2013) Expediting drug development-the FDA's new "breakthrough therapy" designation. N Engl J Med 369:1877-1880

6. D'Hooge J, Heimdal A, Jamal F et al (2000) Regional strain and strain rate measurements by cardiac ultrasound: principles, implementation and limitations. Eur J Echocardiogr 1:154-170

7. Jia C, Olafsson R, Huang S et al (2010) Comparison of 2-D speckle tracking and tissue Doppler imaging in an isolated rabbit heart model. IEEE Trans Ultrason Ferroelectr Freq Control 57:2491-2502

8. Pugh RNH, Murray-Lyon IM, Dawson JL et al (1973) Transection of the oesophagus for bleeding oesophageal varices. Br J Surg 60:646-649

9. Doss W, Esmat G, El-Serafy M et al (2016) Real-life results of sofosbuvir based therapy for egyptian patients with hepatitis $C$ and advanced fibrosiscirrhosis. J Hepatol 64:S772

10. El Raziky M, Gamil M, Ashour MK et al (2016) Simeprevir plus sofosbuvir for eight or 12 weeks in treatment-naïve and treatment-experienced hepatitis C virus genotype 4 patients with or without cirrhosis. J Viral Hepat 24:102-110

11. Mosteller RD (1987) Simplified Calculation of Body Surface Area. N Engl J Med 317:1098

12. Lang RM, Badano LP, Mor-Avi V et al (2015) Recommendations for cardiac chamber quantification by echocardiography in adults: an update from the American Society of Echocardiography and the European Association of Cardiovascular Imaging. J Am Soc Echocardiogr 28:1-39

13. Rudski LG, Lai WW, Afilalo J et al (2010) Guidelines for the echocardiographic assessment of the right heart in adults: a report from the American Society of Echocardiography. J Am Soc Echocardiogr 23:685-713

14. Perk G, Tunick PA, Kronzon I (2007) Non-Doppler two-dimensional strain imaging by echocardiography-from technical considerations to clinical applications. J Am Soc Echocardiogr 20:234-243

15. Ogawa K, Hozumi T, Sugioka K et al (2009) Automated assessment of left atrial function from time-left atrial volume curves using a novel speckle tracking imaging method. J Am Soc Echocardiogr 22:63-69

16. Novo G, Macaione F, Giannitrapani L et al (2018) Subclinical cardiovascular damage in patients with HCV cirrhosis before and after treatment with direct antiviral agents: a prospective study. Aliment Pharmacol Ther 48:740-749

17. Biomy R, Abdelshafy M, Abdelmonem A et al (2017) Effect of chronic hepatitis $C$ virus treatment by combination therapy on cardiovascular system. Clin Med Insights Cardiol 11:117954681771320

18. Durante-Mangoni E, Parrella A, Vitrone M et al (2017) Electrophysiological adverse effects of direct acting antivirals in patients with chronic hepatitis C. J Clin Pharmacol 57:924-930

19. El-Adawy AH, Altonbary AY, Hakim $\mathrm{H}$ et al (2018) Influence of different regimens of direct acting antiviral agents (DAAS) with or without ribavirin used for chronic hepatitis C treatment on the cardiac muscles in Egypt. J Med Res 4:169-173

20. Mazzitelli M, Torti C, Sabatino J et al (2018) Evaluation of cardiac function by global longitudinal strain before and after treatment with sofosbuvir-based regimens in HCV infected patients. BMC Infect Dis 18:518

21. Zaki E, Bahaa El Deen N (2017) Relation of right ventricular dysfunction to the severity of hepatic cirrhosis by different echo modalities using speckletracking echocardiography. Al-Azhar Assiut Med J 15:7-14

22. Hori T, Ogura Y, Onishi Y et al (2016) Systemic hemodynamics in advanced cirrhosis: concerns during perioperative period of liver transplantation. World J Hepatol 8:1047-1060
23. La Villa G, Gentilini P (2008) Hemodynamic alterations in liver cirrhosis. Mol Asp M 29:112-118

24. Kim MY, Baik SK, Lee SS (2010) Hemodynamic alterations in cirrhosis and portal hypertension. Korean J Hepatol 16:347-352

25. Krishnan SM, Dixit NM (2011) Ribavirin-induced anemia in hepatitis C virus patients undergoing combination therapy. PLoS Comput Biol:7e1001072

\section{Publisher's Note}

Springer Nature remains neutral with regard to jurisdictional claims in published maps and institutional affiliations.

\section{Submit your manuscript to a SpringerOpen ${ }^{\circ}$ journal and benefit from:}

- Convenient online submission

- Rigorous peer review

- Open access: articles freely available online

High visibility within the field

- Retaining the copyright to your article

Submit your next manuscript at $\boldsymbol{\nabla}$ springeropen.com 